\title{
Plane Wave Medical Ultrasound Imaging Using Adaptive Beamforming
}

Holfort, Iben Kraglund; Gran, Fredrik; Jensen, Jørgen Arendt

Published in:

Proceedings of 5th IEEE Sensor Array and Multichannel Signal Processing Workshop

Link to article, DOI:

10.1109/SAM.2008.4606874

Publication date:

2008

Document Version

Publisher's PDF, also known as Version of record

Link back to DTU Orbit

Citation (APA):

Holfort, I. K., Gran, F., \& Jensen, J. A. (2008). Plane Wave Medical Ultrasound Imaging Using Adaptive Beamforming. In Proceedings of 5th IEEE Sensor Array and Multichannel Signal Processing Workshop (pp. 288292). IEEE. https://doi.org/10.1109/SAM.2008.4606874

\section{General rights}

Copyright and moral rights for the publications made accessible in the public portal are retained by the authors and/or other copyright owners and it is a condition of accessing publications that users recognise and abide by the legal requirements associated with these rights.

- Users may download and print one copy of any publication from the public portal for the purpose of private study or research.

- You may not further distribute the material or use it for any profit-making activity or commercial gain

- You may freely distribute the URL identifying the publication in the public portal 


\title{
PLANE WAVE MEDICAL ULTRASOUND IMAGING USING ADAPTIVE BEAMFORMING
}

\author{
Iben Kraglund Holfort ${ }^{*}$, Fredrik Gran ${ }^{\dagger}$ and Jørgen Arendt Jensen* \\ ${ }^{*}$ Center for Fast Ultrasound Imaging, Department of Electrical Engineering, \\ Technical University of Denmark, DK-2800 Kgs. Lyngby, Denmark \\ ${ }^{\dagger}$ GN ReSound A/S, Lautrupbjerg 9, DK-2750 Ballerup, Denmark
}

\begin{abstract}
In this paper, the adaptive, minimum variance (MV) beamformer is applied to medical ultrasound imaging. The significant resolution and contrast gain provided by the adaptive, minimum variance (MV) beamformer, introduces the possibility of plane wave (PW) ultrasound imaging. Data is obtained using Field II and a $7 \mathrm{MHz}, 128$-elements, linear array transducer with $\lambda / 2$-spacing. MV is compared to the conventional delay-and-sum (DS) beamformer with Boxcar and Hanning weights. Furthermore, the PW images are compared to the a conventional ultrasound image, obtained from a linear scan sequence. The four approaches, \{Linear Scan, DS Boxcar, DS Hanning, MV\}, have full width at half maximum of $\{0.82,0.71,1.28,0.12\} \mathrm{mm}$ and peak side-lobe levels of $\{-40.1,-16.8,-34.4,-57.0\} \mathrm{dB}$.
\end{abstract}

\section{INTRODUCTION}

A conventional ultrasound image is formed from several focused ultrasound transmissions, so that each scan line in the image is obtained from an additional, focused ultrasound transmission. To cover the entire image region, the aperture is slid along the scan plane.

An alternative approach is to use a single unfocused transmission, also referred to as a plane wave transmission. In this way, the entire image region is covered in a single transmission. However, this approach traditionally provides images with poor contrast and resolution due to the limitations of the conventional, delay-and-sum (DS) beamformer.

For decades data-dependent, adaptive beamformers have been used in other fields of array signal processing, e.g. sonar and radar. Whereas the conventional beamformer is a passive process using predefined, fixed, data-independent (apodization) weights, the adaptive beamformer actively updates a set of new apodization weights for each point in the image. These apodization weights are dependent on the input data. One of the widely used methods was originally

This work was supported by grant 26-04-0024 from the Danish Science Foundation, grant 274-05-0327 from the Danish Research Agency, the Radio-parts foundation and by B-K Medical ApS, Denmark. introduced by Capon in 1969 [1].

Recently, the application of adaptive beamforming to the field of medical ultrasound imaging has been an area of increased interest. In recent literature [2, 3, 4, 5, 6], adaptive beamformers have been applied to medical ultrasound imaging with significant improvements in terms of lateral resolution and contrast.

Previously, an approach for near-field beamforming of broad-band ultrasound data has been proposed [6]. This approach is implemented in the frequency domain, and it provides a set of adapted, complex apodization weights for each frequency sub-band.

The purpose of this paper is to apply the proposed beamformer to ultrasound data obtained from a plane wave (PW) transmission. The performance of the proposed beamformer is compared to the DS beamformer using Boxcar and Hanning weights. Furthermore, the resulting image from the PW sequence is compared to an image obtained using a conventional ultrasound, focused transmission sequence.

\section{METHOD}

As in conventional beamforming, the sensor signals are presteered, so that each scan line is dynamically focused. Considering a linear array transducer with $M$ sensor elements, the $m$ th dynamically focused sensor signal along the $\ell$ th scan line is given by

$$
y_{m, \ell}(z)=s\left(\tau_{m, \ell}(z)\right)
$$

for $m=0,1, \ldots, M-1$ and $\ell=0,1, \ldots, L-1$, where $z$ denotes the spatial position along the $\ell$ th scan line, $s(t)$ is the received waveform. The delay line, $\tau_{m, \ell}(z)$, is found from the propagation path of the sound waves from the transmitting element to the focal point and returning to the receiving element, and is given by

$$
\tau_{m, \ell}(z)=\frac{\left\|\vec{r}^{(x m t)}-\vec{r}_{\ell}(z)\right\|+\left\|\vec{r}_{m}^{(r c v)}-\vec{r}_{\ell}(z)\right\|}{c}
$$

where $\vec{r}^{(x m t)}$ and $\vec{r}_{m}^{(r c v)}$ are the spatial positions of the transmitting and the $m$ th receiving sensor elements, $\vec{r}_{\ell}(z)$ 
denotes the focal point, and $c$ is the speed of sound. The output of the beamformer is given by the weighted sum of the dynamically focused scan lines.

\subsection{Sub-Band Beamforming}

The MV beamformer [1] is originally developed for narrowband applications. Applying MV to broad-band ultrasound data, the sensor signals are divided into sub-bands using the short-time Fourier transform. For each point, $z_{0}$, along the $\ell$ th scan line, the Fourier transform is applied on a segment of the sensor signals. The $m$ th segmented sensor signal is given by

$$
\tilde{y}_{m, \ell}\left(z, z_{0}\right)=y_{m, \ell}\left(z+z_{0}\right), \quad z \in[-Z / 2 ; Z / 2],
$$

where $Z$ is the size of the segment. For the given point, $z_{0}$, the beamformer output for each spatial frequency sub-band, $k$, is given by

$$
B_{\ell}\left(k, z_{0}\right)=\sum_{m=0}^{M-1} w_{m, \ell}^{*}\left(k, z_{0}\right) Y_{m, \ell}\left(k, z_{0}\right)
$$

where $Y_{m, \ell}\left(k, z_{0}\right)$ is the Fourier transform of the $m$ th segmented sensor signal, $\tilde{y}_{m, \ell}\left(z, z_{0}\right)$, given in (3), and $\{\cdot\}^{*} \mathrm{de}-$ notes the complex conjugate. By defining the vectors

$$
\begin{aligned}
& \mathbf{w}_{\ell}\left(k, z_{0}\right)=\left(\begin{array}{llll}
w_{0, \ell}\left(k, z_{0}\right) & w_{1, \ell}\left(k, z_{0}\right) & \cdots & w_{M-1, \ell}\left(k, z_{0}\right)
\end{array}\right)^{T} \\
& \mathbf{Y}_{\ell}\left(k, z_{0}\right)=\left(\begin{array}{llll}
Y_{0, \ell}\left(k, z_{0}\right) & Y_{1, \ell}\left(k, z_{0}\right) & \cdots & Y_{M-1, \ell}\left(k, z_{0}\right)
\end{array}\right)^{T}
\end{aligned}
$$

the beamformer output (4) rewrites into

$$
B_{\ell}\left(k, z_{0}\right)=\mathbf{w}_{\ell}\left(k, z_{0}\right)^{H} \mathbf{Y}_{\ell}\left(k, z_{0}\right),
$$

where the superscripts, $\{\cdot\}^{T}$ and $\{\cdot\}^{H}$, denote the non-conjugate and the conjugate transpose, respectively. Note that the sub-band division provides the possibility of weighting both each sub-band and each point differently.

\subsection{Minimum Variance Beamforming}

The adaptive beamformer uses a set of apodization weights, which are dependent on the frequency content of the specific sensor signals. The MV beamformer continuously updates the weights, so that the variance (or power) of the beamformer output is minimized, while the response from the focus point is passed without distortion. The power of the beamformer output is given by

$$
\begin{aligned}
\mathcal{P}_{\ell}\left(k, z_{0}\right) & =\mathcal{E}\left\{\left|B_{\ell}\left(k, z_{0}\right)\right|^{2}\right\} \\
& =\mathcal{E}\left\{\left|\mathbf{w}\left(k, z_{0}\right)^{H} \mathbf{Y}_{\ell}\left(k, z_{0}\right)\right|^{2}\right\} \\
& =\mathbf{w}_{\ell}\left(k, z_{0}\right)^{H} \mathbf{R}_{\ell}\left(k, z_{0}\right) \mathbf{w}_{\ell}\left(k, z_{0}\right),
\end{aligned}
$$

where $\mathcal{E}\{\cdot\}$ denotes the expectation value, and $\mathbf{R}_{\ell}\left(k, z_{0}\right)$ is the covariance matrix given by

$$
\mathbf{R}_{\ell}\left(k, z_{0}\right)=\mathcal{E}\left\{\mathbf{Y}_{\ell}\left(k, z_{0}\right) \mathbf{Y}_{\ell}\left(k, z_{0}\right)^{H}\right\}
$$

Mathematically, the MV beamformer is expressed as [1]

$$
\begin{gathered}
\min _{\mathbf{w}_{\ell}\left(k, z_{0}\right)} \mathbf{w}_{\ell}\left(k, z_{0}\right)^{H} \mathbf{R}_{\ell}\left(k, z_{0}\right) \mathbf{w}_{\ell}\left(k, z_{0}\right) \\
\quad \text { subject to } \mathbf{w}_{\ell}\left(k, z_{0}\right)^{H} \mathbf{e}\left(k, z_{0}\right)=1,
\end{gathered}
$$

where $\mathbf{e}\left(k, z_{0}\right)$ is the so-called steering vector, which characterizes the response from the focus point.

The solution to the optimization problem (10) can be found in a single iteration using Lagrangian multiplier theory as [1]

$$
\mathbf{w}_{\ell}\left(k, z_{0}\right)=\frac{\mathbf{R}_{\ell}\left(k, z_{0}\right)^{-1} \mathbf{e}\left(k, z_{0}\right)}{\mathbf{e}\left(k, z_{0}\right)^{H} \mathbf{R}_{\ell}\left(k, z_{0}\right)^{-1} \mathbf{e}\left(k, z_{0}\right)},
$$

provided that $\mathbf{R}_{\ell}\left(k, z_{0}\right)^{-1}$ exists. Due to presteering and sub-band division, the response from the focus point will resemble a plane wave incident directly onto the array. Thus, the steering vector is constant across the array and independent on the frequency, and it simply becomes a $M \times 1$-vector of ones.

\subsection{Covariance Matrix Estimation}

In real applications, the covariance matrix is unknown and must be replaced by the sample covariance matrix, which is estimated from the data. To estimate the sample covariance matrix a number of realizations of data is required. In this paper, these realizations are obtained by dividing the data from a single acquisition into a number of subgroups. This follows the spatial smoothing approach suggested in [7].

As illustrated in Fig. 1, the spatially smoothed covariance matrix estimate is obtained by dividing the array into $P$ overlapping subarrays of size $M_{p} \leq \frac{M}{2}$. For each subarray, a sub-covariance matrix is estimated, and these are averaged across the array. The covariance matrix estimate

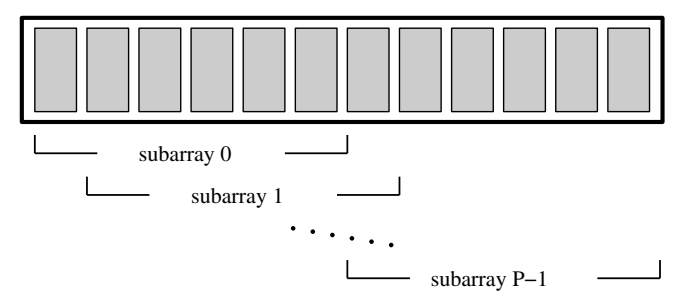

Fig. 1. Spatial smoothing. The array is divided into $P$ overlapping subarrays, and the covariance matrix is averaged across the array. 
can be expressed as

$$
\mathbf{R}_{\ell}\left(k, z_{0}\right)=\frac{1}{P} \sum_{p=0}^{P-1} \mathbf{G}_{p, \ell}\left(k, z_{0}\right) \mathbf{G}_{p, \ell}\left(k, z_{0}\right)^{H}
$$

where $\mathbf{G}_{p, \ell}\left(k, z_{0}\right)$ denotes the $p$ th subarray given by

$$
\mathbf{G}_{p, \ell}\left(k, z_{0}\right)=\left[\begin{array}{c}
Y_{p, \ell}\left(k, z_{0}\right) \\
Y_{p+1, \ell}\left(k, z_{0}\right) \\
\vdots \\
Y_{p+M_{p}-1, \ell}\left(k, z_{0}\right)
\end{array}\right]
$$

for $p=0,1, \ldots, P-1$. Note that this reduces the dimension of the covariance matrix, and thus the number of weights will be reduced correspondingly. The reduced weight vector, $\widetilde{\mathbf{w}}_{\ell}\left(k, z_{0}\right)$, is applied to the data by averaging over the $P$ subarrays, which is expressed as

$$
\mathbf{R}_{\ell}\left(k, z_{0}\right)=\widetilde{\mathbf{w}}_{\ell}\left(k, z_{0}\right)^{H} \frac{1}{P} \sum_{p=0}^{P-1} \mathbf{G}_{p, \ell}\left(k, z_{0}\right) .
$$

Note that the reduced dimension also influences the resolution due to the inherent compromise between the width of the array and the achievable resolution [8].

\section{RESULTS}

The MV beamformer is applied to simulated medical ultrasound data, obtained using the ultrasound simulation program, Field II [9, 10]. For the simulations, a linear array transducer with a center frequency of $f_{0}=7 \mathrm{MHz}, \lambda / 2$ spacing and $M=128$ sensor elements was used. Data of 13 point targets is obtained using two different transmission sequences; a conventional, linear scan, see Sec. 3.1, and a PW transmission, see Sec. 3.2.

The MV beamformer is implemented in the frequency domain using the short-time Fourier transform with a segment size corresponding to the length of the excitation pulse convoluted with the two-way impulse response of the transducer. A subarray size of $M_{p}=\frac{M}{4}=32$ was used, and the signal-to-noise ratio was $60 \mathrm{~dB}$.

\subsection{Linear Scan}

Conventionally ultrasound imaging is carried out by transmitting several focused ultrasound beams, e.g. using a linear scan sequence as illustrated in Fig. 2(a). The transmitted pulses from each of the transducer elements are delayed so that they add up constructively in phase in a single point in space, the transmit focus.

To cover the entire image region, a large array is used and a part of the aperture is slid electronically along the scan plane. The image in Fig. 3(a) is obtained with a sliding

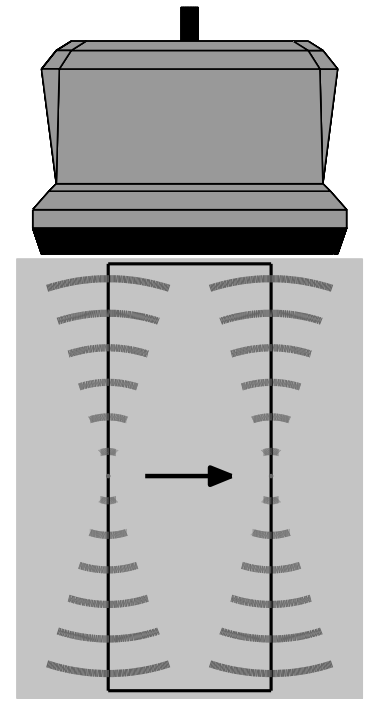

(a) Linear Scan

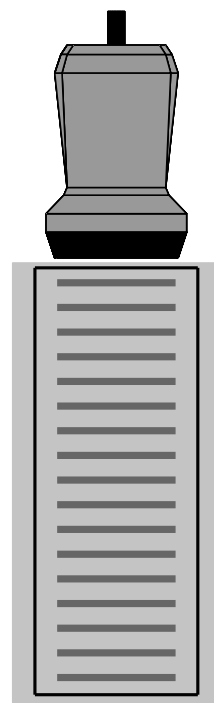

(b) Plane Wave
Fig. 2. Illustration of scan sequences, the transducer is shown in the top and the image region is indicated by the black lines. (a) A linear scan is carried out using a large array and transmitting several focused beams, while sliding the active part of the aperture along the scan plane. (b) Using a plane wave transmission the entire image region is obtained from a single transmission. Courtesy of Dr. J. Udesen.

aperture size of 128 elements and a fixed transmit focus at a depth of $z=45 \mathrm{~mm}$. The response is beamformed using DS and dynamic Hanning apodization weights with $f / 2$.

\subsection{Plane Wave Transmission}

A plane wave (PW) transmission can be achieved by using all elements are as both the transmitting and receiving aperture. In this way, the entire image region can be covered in a single transmission, as illustrated in Fig. 2(b). Note that when using PW, the propagation path from the transmitting element to the current image point, $\vec{r}_{\ell}\left(z_{0}\right)$, in (2) simply becomes the axial distance, $z_{0}$, from the array to the image point

$$
\left\|\vec{r}^{(x m t)}-\vec{r}_{\ell}\left(z_{0}\right)\right\|=z_{0}
$$

which is independent on the scan line, $\ell$.

The MV beamformed response is compared to DS using Boxcar and Hanning weights. The beamformed responses are seen in Fig. 3(b)-(d) with the lateral variation at $z=$ $\{40,45\} \mathrm{mm}$ shown in Fig. 4. The resolution and contrast are quantified by the Full Width at Half Maximum (FWHM) and the Peak-Side-lobe Level (PSL), which is defined as the peak of the first side-lobe. These are given in Table 1. 


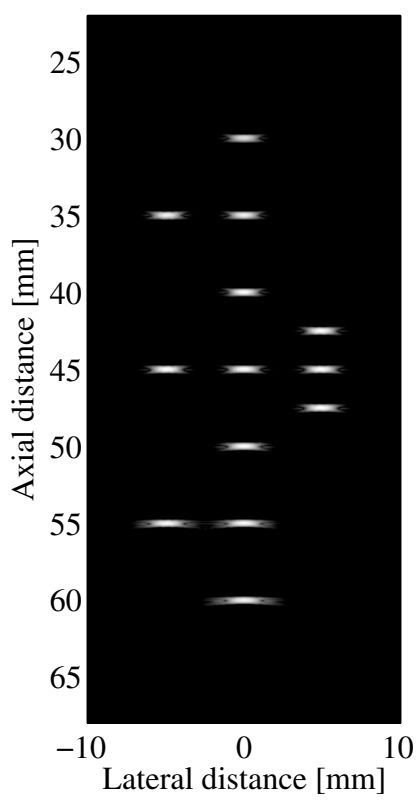

(a) Linear Scan

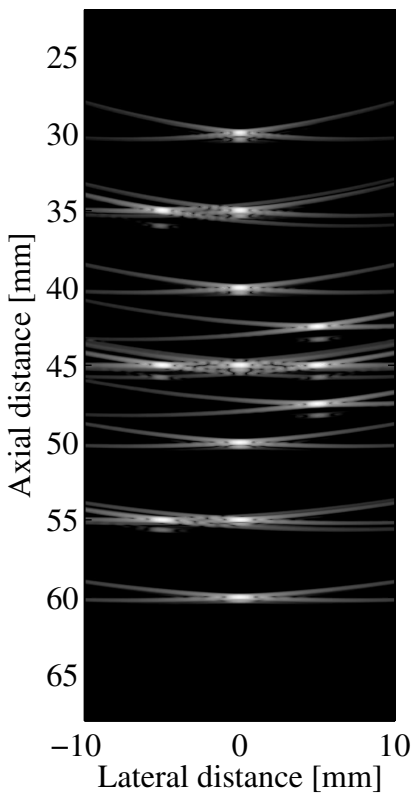

(b) DS, Boxcar

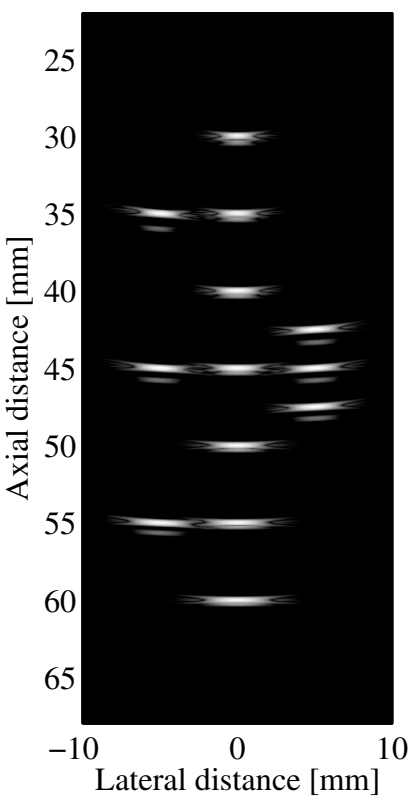

(c) DS, Hanning

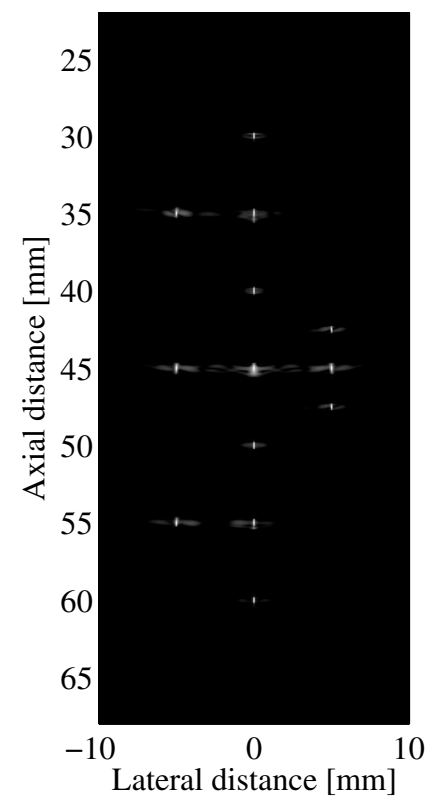

(d) MV

Fig. 3. Beamformed responses of the 13 point targets. (a) Linear scan, DS with dynamic apodization using Hanning weights. (b)-(d) Plane wave transmission. All images are shown with a dynamic range of $50 \mathrm{~dB}$.

\begin{tabular}{lccc} 
& PSL & \multicolumn{2}{c}{ FWHM } \\
\hline Linear Scan & $-40.1 \mathrm{~dB}$ & $0.82 \mathrm{~mm}$ & $3.71 \lambda$ \\
DS, Boxcar & $-16.8 \mathrm{~dB}$ & $0.71 \mathrm{~mm}$ & $3.23 \lambda$ \\
DS, Hanning & $-34.4 \mathrm{~dB}$ & $1.28 \mathrm{~mm}$ & $5.81 \lambda$ \\
MV & $-57.0 \mathrm{~dB}$ & $0.12 \mathrm{~mm}$ & $0.53 \lambda$ \\
\hline$\lambda=c / f_{0}=220 \mu \mathrm{m}$ & &
\end{tabular}

Table 1. Full Width at Half Maximum (FWHM) and Peakside-lobe level (PSL) for the beamformed responses found at a depth of $z=40 \mathrm{~mm}$.

The PW data is obtained with Boxcar apodization both in transmit and receive. This provides unwanted side-lobes below the point targets, as seen in Fig. 3(b)-(c). These could have been removed by using a transmit apodization, such as a Tukey window with $70 \%$ tapering as in [11]. However, this has not been done, to demonstrate that the MV beamformer successes in eliminating these, as seen in Fig. 3(d).

Due to the focused transmissions and the dynamic Hanning apodization, the linear scan sequence provides a more homogeneous image than the unfocused PW transmissions. The resolution and contrast of the PW data are degraded and the images are not acceptable for ultrasound imaging.

The MV beamformer provides an increase of the resolution and contrast, as seen in Fig. 3(d) and Fig. 4. Even compared to the linear scan image, the MV beamformer provides a significant increase of the FWHM and PSL. From
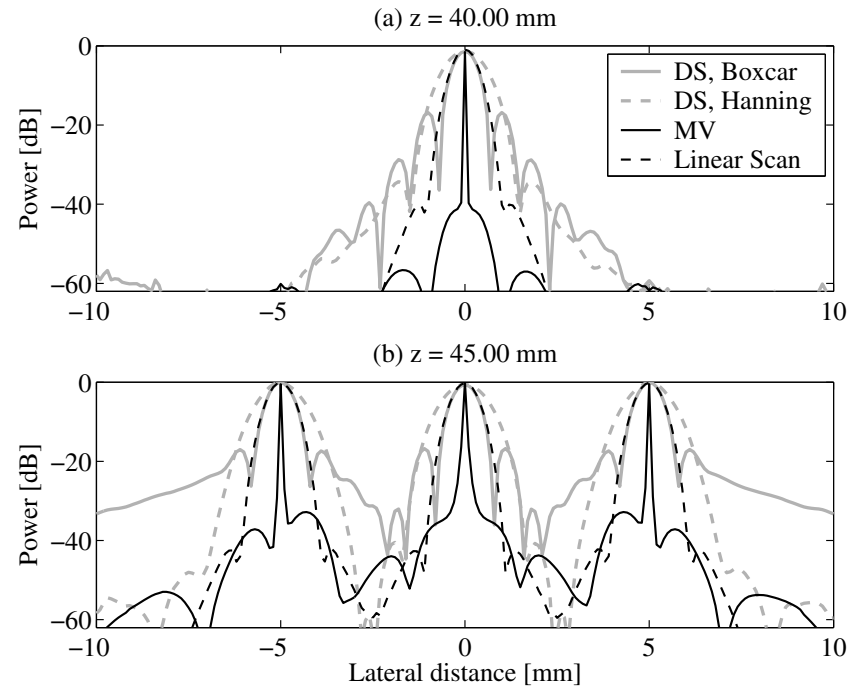

Fig. 4. Lateral variation at a depth of $z=\{40,45\} \mathrm{mm}$ of the beamformed responses shown in Fig. 3(a)-(d).

this investigation, it is seen that the increase of resolution and contrast, provided by the MV beamformer, yields the possibility of high resolution and contrast $\mathrm{PW}$ imaging, where an image region can be covered in a single transmission. 


\section{CONCLUSIONS}

The adaptive MV beamformer has been applied to simulated ultrasound data obtained from a PW transmission. The performance of the MV beamformer is compared to the DS beamformer using Boxcar and Hanning weights. The results have shown that the MV beamformer provides a significant increase in resolution and contrast compared to the DS beamformer. Furthermore, the MV beamformed PW response is compared to data obtained from a conventional ultrasound sequence, where several focused transmission are used. These results have shown that the significant increase in resolution and contrast, provided by the MV beamformer, yields the possibility of PW medical ultrasound imaging with increased resolution and contrast.

\section{REFERENCES}

[1] J. Capon, "High-Resolution Frequency-Wavenumber Spectrum Analysis," Proc. IEEE, vol. 57, no. 8, pp. 1408-1418, August 1969

[2] J. A. Mann and W. F. Walker, "A Constrained Adaptive Beamformer for Medical Ultrasound: Initial Results," in Proc. IEEE Ultrason. Symp., Oct. 2002, vol. 2, pp. 18071810.

[3] M. Sasso and C. Cohen-Bacrie, "Medical Ultrasound Imaging Using the Fully Adaptive Beamformer," in Proc. IEEE Int. Conf. Acous., Speech, Sig. Pro., March 2005, vol. 2, pp. 489-492.

[4] Z. Wang, J. Li, and R. Wu, "Time-Delay- and TimeReversal-Based Robust Capon Beamformers for Ultrasound Imaging," IEEE Trans. Med. Imag., vol. 24, no. 10, pp. 1301322, Oct. 2007.

[5] J.-F. Synnevåg, A. Austeng, and S. Holm, "Adaptive Beamforming Applied to Medical Ultrasound Imaging," IEEE Trans. Ultrason., Ferroelec., Freq. Contr., vol. 54, no. 8, pp. 1606-1613, Aug. 2007.

[6] I. K. Holfort, F. Gran, and J. A. Jensen, "Minimum Variance Beamforming for High Frame-Rate Ultrasound Imaging," in Proc. IEEE Ultrason. Symp., Oct. 2007, pp. 1541-1544.

[7] T.-J. Shan and T. Kailath, "Adaptive Beamforming for Coherent Signals and Interference," IEEE Trans. Acous., Speech, Sig. Pro., vol. 33, no. 3, pp. 527-536, June 1985.

[8] D. H. Johnson and D. E. Dudgeon, Array Signal Processing: Concepts and Techniques, Prentice-Hall., Englewood Cliffs, New Jersey, 1993.

[9] J. A. Jensen and N. B. Svendsen, "Calculation of pressure fields from arbitrarily shaped, apodized, and excited ultrasound transducers," IEEE Trans. Ultrason., Ferroelec., Freq. Contr., vol. 39, pp. 262-267, 1992.

[10] J. A. Jensen, "Field: A program for simulating ultrasound systems," Med. Biol. Eng. Comp., vol. 10th Nordic-Baltic Conference on Biomedical Imaging, Vol. 4, Supplement 1, Part 1, pp. 351-353, 1996b.
[11] J. Udesen, F. Gran, and J. A. Jensen, "Fast color flow mode imaging using plane wave excitation and temporal encoding," in Proc. SPIE - Progress in biomedical optics and imaging, 2005, vol. 5750, pp. 427-436. 